\title{
Cutaneous mucormycosis mimicking pancreatic panniculitis
}

\author{
Pedro Miguel Garrido $^{1}$ @ | Rita Pimenta ${ }^{1}$ | Isabel Viana $^{2}$ | Heinz Kutzner ${ }^{3}$ | \\ Paulo Filipe $^{1,4,5}$ | Luís Soares-Almeida ${ }^{1,4,5}$ \\ ${ }^{1}$ Dermatology Department, Hospital de Santa Maria, Centro Hospitalar Universitário Lisboa Norte (CHULN), Lisbon, Portugal \\ ${ }^{2}$ Dermatology Department, Hospital Egas Moniz, Centro Hospitalar de Lisboa Ocidental (CHLO), Lisbon, Portugal \\ ${ }^{3}$ Dermatopathologie Friedrichshafen, Friedrichshafen, Germany \\ ${ }^{4}$ Dermatology Universitary Clinic, Faculty of Medicine, University of Lisbon, Lisbon, Portugal \\ ${ }^{5}$ Dermatology Research Unit, Instituto de Medicina Molecular, University of Lisbon, Lisbon, Portugal \\ Correspondence \\ Garrido PM; Adress: Centro Hospitalar Lisboa Norte EPE, Av. Prof. Egas Moniz, Lisboa 1649-035, Portugal. \\ Email: pedro.mi.garrido@gmail.com
}

KEYWORDS: mucormycosis, pancreatic panniculitis, panniculitis.

\section{1 | INTRODUCTION}

Mucormycosis is an uncommon, life-threatening infection caused by opportunistic fungi included in the Mucorales order, which mostly affects immunosuppressed patients. ${ }^{1}$ Mucormycosis can exhibit different clinical presentations depending on the involved organ, including pulmonary, gastrointestinal, rhinocerebral, cutaneous, and disseminated infections. ${ }^{1}$

Primary cutaneous mucormycosis results from percutaneous inoculation of fungal spores, usually following thermal burns or trauma. A secondary form results from hematogenous dissemination and affects predominantly immunosuppressed patients. ${ }^{1}$

Although nonspecific, most patients with cutaneous mucormycosis will present with a necrotic ulcer, nodule or plaque with surrounding erythema and edema. ${ }^{1}$ The histopathological examination shows a variable appearance. $^{2}$

We report a case of primary cutaneous mucormycosis which most striking histopathologic feature was groups of necrotic adipocytes lacking nuclei, with a finely granular and basophilic hue within the cytoplasm, resembling pancreatic panniculitis.

\section{1 | Case presentation}

A 67-year-old Caucasian woman presented to our dermatology clinic with a two-week history of a painful, large necrotic ulcer overlying an erythematous and violaceous patch on the left leg. (Figure 1). Four months before, she was diagnosed with renal cell carcinoma with lung and brain metastases, and was being treated with sunitinib. She was also undergoing whole-brain radiotherapy in combination with dexamethasone $4 \mathrm{mg}$ twice daily. She denied local trauma or fever.
An incisional biopsy at the edge of the lesion revealed a predominantly lobular panniculitis, with groups of necrotic adipocytes lacking nuclei, with a finely granular and basophilic hue within the cytoplasm due to saponification (Figure 2). These findings closely resembled the so-called ghost adipocytes of pancreatic panniculitis. However, serum amylase and lipase levels were not elevated, she did not present abdominal pain and had no prior history of pancreatic disease. Additionally, a predominantly neutrophilic infiltrate and lipophagic granulomas were seen at the periphery of necrotic fat lobules. No needleshaped refractile crystals were seen within necrotic adipocytes.

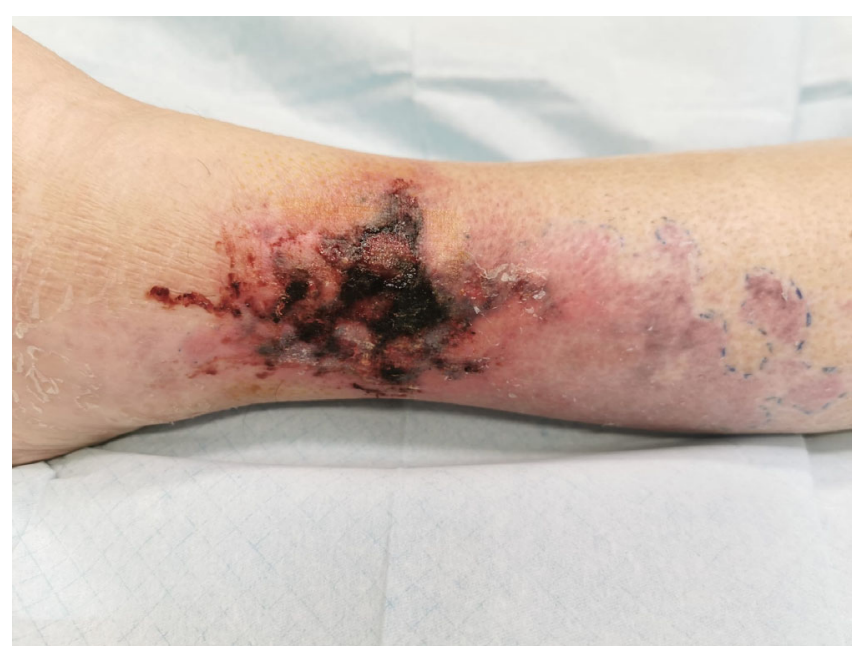

FIGURE 1 Clinical image showing a large $7 \mathrm{~cm}$ stellate necrotic ulcer overlying an erythematous and violaceous patch on the left lateral distal lower leg 
(A)

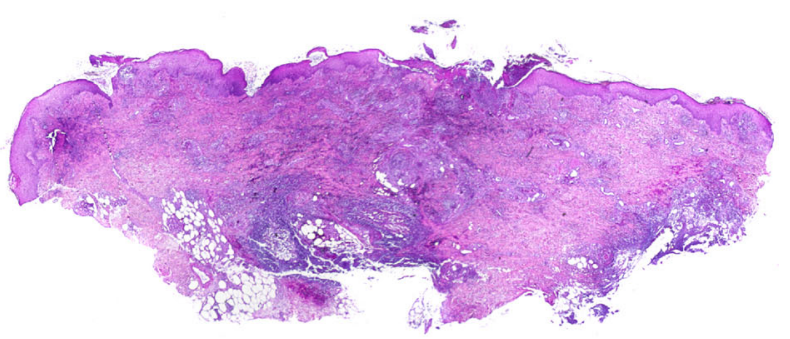

(C)

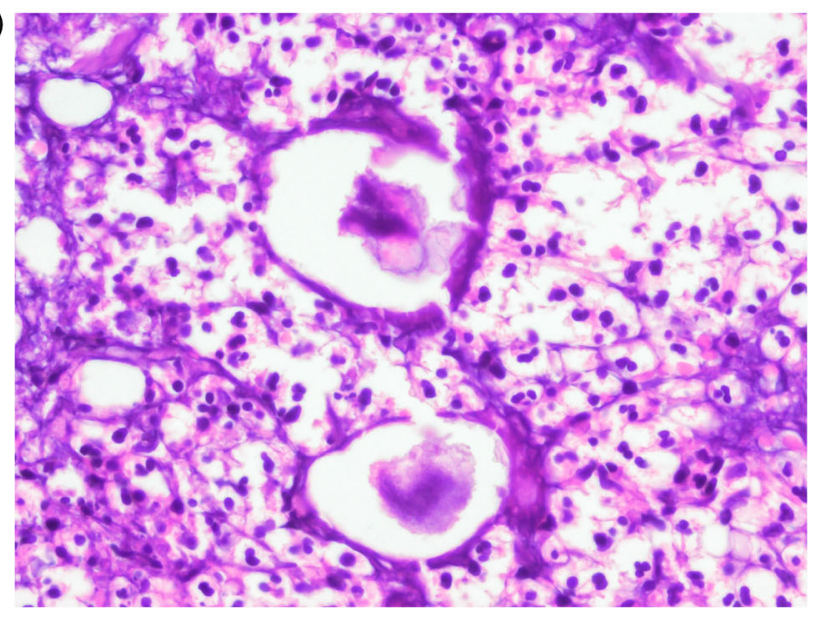

(E)

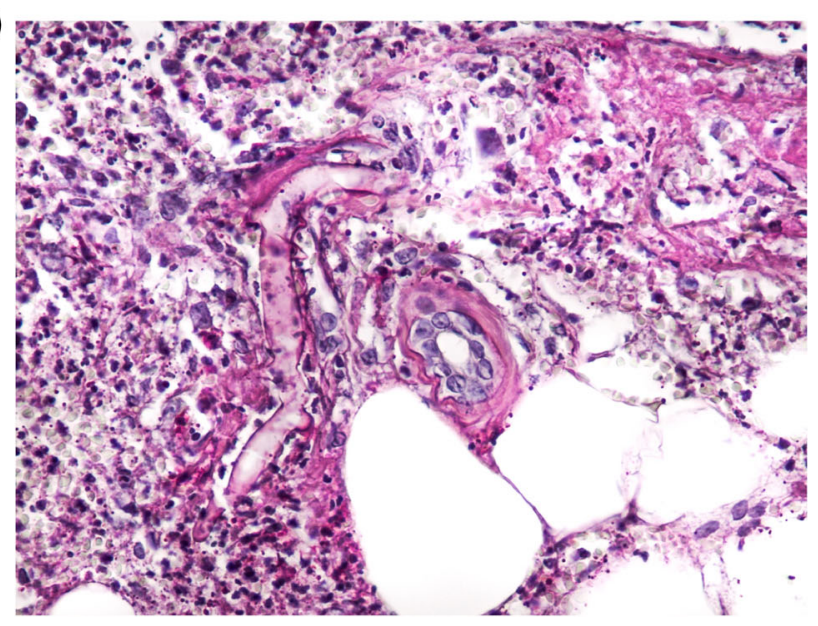

(B)

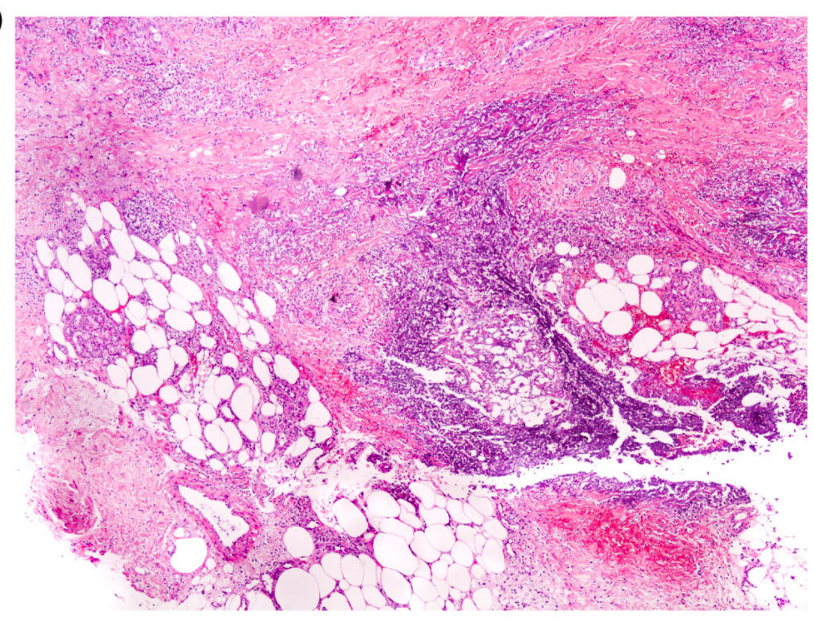

(D)

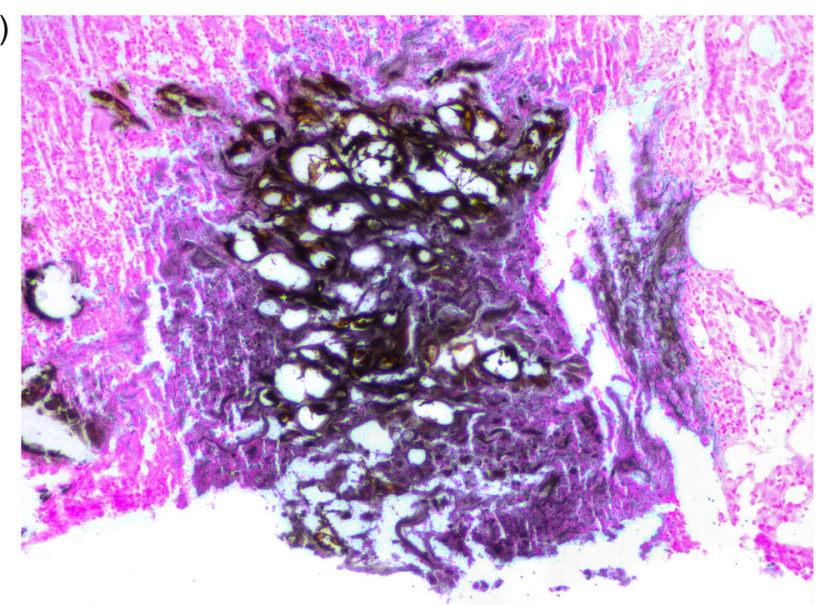

FIGURE 2 Histopathology features (A) Scanning magnification shows a predominantly lobular panniculitis. (B) Large areas of necrotic adipocytes, with a dense inflammatory infiltrate and red cell extravasation. (C) Necrotic adipocytes lacking nuclei, with a finely granular and basophilic hue within the cytoplasm. A predominantly neutrophilic infiltrate is present at the periphery of necrotic fat lobules. (D) Large areas of calcification. (E) Broad, thick-walled, non-septate hyphae scattered throughout necrotic areas. (A-C: Hematoxylin-eosin stain; original magnifications: 5x, 40x, 400x; D: von-Kossa stain; original magnification: 100x; E: Periodic acid-Schiff stain: original magnification: 400x)

Numerous broad, thick-walled, non-septate hyphae scattered throughout necrotic areas were highlighted with periodic acid-Schiff (PAS) and Grocott stains. These hyphae seemed to branch in a rightangle. We did not find any fungal hyphae within the wall and lumina of blood vessels and there was no evidence of congestion or thrombosis. The diagnosis of a panniculitis secondary to fungal infection was established.

A skin polymerase chain reaction (PCR) assay detected DNA from Rhizopus oryzae. Blood cultures did not isolate any microorganism and the galactomannan antigen assay was negative.
The patient underwent surgical debridement, but died after one week. The cause of her death was attributed to advanced neoplastic disease.

\section{2 | DISCUSSION}

Cutaneous mucormycosis is a deep fungal infection of the skin that can present different histopathological patterns. Most patients have typical features of infective panniculitis, with suppurative granulomas 
within the lobule and a neutrophilic infiltrate. ${ }^{2}$ Requena et al described a series of 13 patients with cutaneous mucormycosis in which histopathologic features mimicked pancreatic panniculitis and gouty panniculitis. ${ }^{3}$ As in the cases reported, we observed a lobular, calcifying panniculitis with ghost adipocytes that are the hallmark of pancreatic panniculitis.

Pancreatic panniculitis is a lobular necrotizing panniculitis, featuring ghost-like adipocytes with thick shadowy walls and no nuclei as well as granular basophilic material within and around necrotic adipocytes, due to saponification of free fatty acids by calcium salts. ${ }^{4}$ The pathogenesis of this disorder is explained by distant action of systemically released pancreatic enzymes, mostly lipase, that cause lipolysis and fat necrosis with consecutive inflammatory reaction. ${ }^{4}$ Requena et al suggested that the pathogenesis of cutaneous mucormycosis presenting as a pancreatic panniculitis is likely to be related to the local effect of the lipase produced by Mucoraceae fungi. ${ }^{3}$ Dystrophic calcification in ghost adipocytes in cutaneous mucormycosis likely results from hydrolytic action of lipase on fat followed by calcium deposition, a process known as saponification. ${ }^{3}$

Previous reports described overlap cases where both ghost adipocytes and needle-shaped refractile crystals were seen within necrotic adipocytes. ${ }^{3,5,6}$ As with ghost adipocytes, urate crystal production in cutaneous mucormycosis may be caused by enzymatic substances released from Mucoraceae fungi. These fungi are thought to produce oxalic acid that act on the purine contents of necrotic adipocytes thereby resulting in the formation of calcium oxalate monohydrate crystals. ${ }^{3}$ However, we did not find intracellular crystals resembling urate crystals found in gouty panniculitis.

The increase in serum amylase and/or lipase levels is a welldescribed side effect of tyrosine kinase inhibitors, including sunitinib. Although rare, acute pancreatitis has also been reported, mostly in patients being treated for metastatic renal cell carcinoma. ${ }^{7}$ The presence of numerous fungal hyphae scattered through necrotic areas allowed the histopathologic differential diagnosis with pancreatic panniculitis. Furthermore, our patient had no prior history pancreatic disease, did not present abdominal pain and serum lipase and amylase were not elevated.

The differential diagnosis also included other fungal-related panniculitis, in particular cutaneous aspergillosis, as this microorganism is able to secrete extracellular lipase and therefore can mimic pancreatic panniculitis. ${ }^{5}$ The $90^{\circ}$ branching was suggestive of mucormycosis and skin PCR assay confirmed the diagnosis.

We present a case of primary cutaneous mucormycosis without inciting trauma, in a patient with a metastatic malignant neoplasm who was being treated with a tyrosine kinase inhibitor. Although most cases of primary cutaneous mucormycosis result from percutaneous inoculation of fungal spores following thermal burns or trauma, this condition has also been reported in immunocompromised patients without any precipitating factor. ${ }^{8}$

In our case, the diagnosis of cutaneous mucormycosis was only considered after the histopathologic finding of fungal hyphae scattered through subcutaneous tissue. Skin PCR assay allowed fungus identification. The patient presented a rapidly progressive and aggressive clinical course, unresponsive to surgical debridement and died before initiating any antifungal drug. Cutaneous mucormycosis is a serious infection, with a high overall mortality. ${ }^{2}$ The diagnosis of cutaneous mucormycosis is challenging and a high clinical awareness may improve the prognosis, as early diagnosis and prompt management are crucial to prevent fatal outcome.

\section{3 | CONCLUSION}

Cutaneous mucormycosis is an emerging fungal infection that must be considered in the differential diagnosis of a calcifying panniculitis, particularly in immunocompromised patients. Although ghost adipocytes are a classic hallmark of pancreatic panniculitis, an infectious source should be considered in their presence.

\section{CONFLICT OF INTEREST}

The authors declare not having any conflict of interests.

\section{ORCID}

Pedro Miguel Garrido (D) https://orcid.org/0000-0002-1048-3056

\section{REFERENCES}

1. Shields BE, Rosenbach $M$, Brown-Joel Z, Berger AP, Ford BA, Wanat KA. Angioinvasive fungal infections impacting the skin: background, epidemiology, and clinical presentation. J Am Acad Dermatol. 2019;80(4):869-880.e5.

2. Berger AP, Ford BA, Brown-Joel Z, Shields BE, Rosenbach $M$, Wanat KA. Angioinvasive fungal infections impacting the skin: diagnosis, management, and complications. J Am Acad Dermatol. 2019;80(4): 883.e2-898.e2

3. Requena L, Sitthinamsuwan P, Santonja C, et al. Cutaneous and mucosal mucormycosis mimicking pancreatic panniculitis and gouty panniculitis. J Am Acad Dermatol. 2012;66(6):975-984.

4. Requena L, Sánchez Yus E. Panniculitis. Part II. Mostly lobular panniculitis. J Am Acad Dermatol. 2001;45(3):325-364.

5. Colmenero I, Alonso-Sanz M, Casco F, Hernández-Martín A, Torrelo A. Cutaneous aspergillosis mimicking pancreatic and gouty panniculitis. J Am Acad Dermatol. 2012;67(4):789-791.

6. Hawkes JE, Florell SR, Wada DA. Chronic, painful, nonhealing ulcer on the right arm following minor trauma. JAMA Dermatol. 2015;151(7): 787-788.

7. Sevin A, Chen A, Atkinson B. Tyrosine kinase inhibitor induced pancreatitis. J Oncol Pharm Pract. 2013;19(3):257-260.

8. Lenane $\mathrm{P}$, Keane $\mathrm{CO}$, Loughlin SO. Mucormycosis infection presenting as a non-healing ulcer in an immunocompromised patient. Clin Exp Dermatol. 2003 Mar;28(2):157-159.

How to cite this article: Garrido PM, Pimenta R, Viana I, Kutzner H, Filipe P, Soares-Almeida L. Cutaneous mucormycosis mimicking pancreatic panniculitis. J Cutan Pathol. 2021;48(8):1007-1009. https://doi.org/10.1111/cup. 13763 